\title{
Slicing and Dicing a Newspaper Corpus for Historical Ecology Research
}

\author{
Marieke van $\operatorname{Erp}^{1(\otimes)}$, Jesse de Does ${ }^{2}$, Katrien Depuydt ${ }^{2}$, Rob Lenders $^{3}$, \\ and Thomas van Goethem ${ }^{3}$ \\ 1 DHLab, KNAW Humanities Cluster, Amsterdam, Netherlands \\ marieke.van.erp@dh.huc.knaw.nl \\ 2 Instituut voor de Nederlandse Taal, Leiden, Netherlands \\ \{jesse.dedoes, katrien.depuydt\}@ivdnt.org \\ 3 Radboud University Nijmegen, Nijmegen, Netherlands \\ $\{r$.lenders, tgoethem $\}$ @science.ru.nl
}

\begin{abstract}
Historical newspapers are a novel source of information for historical ecologists to study the interactions between humans and animals through time and space. Newspaper archives are particularly interesting to analyse because of their breadth and depth. However, the size and the occasional noisiness of such archives also brings difficulties, as manual analysis is impossible. In this paper, we present experiments and results on automatic query expansion and categorisation for the perception of animal species between 1800 and 1940. For query expansion and to the manual annotation process, we used lexicons. For the categorisation we trained a Support Vector Machine model. Our results indicate that we can distinguish newspaper articles that are about animal species from those that are not with an $\mathrm{F}_{1}$ of 0.92 and the subcategorisation of the different types of newspapers on animals up to $0.84 \mathrm{~F}_{1}$.
\end{abstract}

Keywords: Natural language processing $\cdot$ Lexicology $\cdot$ Humanities Historical ecology · Digital libraries

\section{Introduction}

Digital newspaper archives are becoming a widely used resource for humanities and social sciences researchers $[5,18,23]$. However, making sense of the large amounts of data that these archives contain requires computational methods, which are often not (yet) part of the standard toolkit of humanities and social sciences researchers. In this work, we present an interdisciplinary collaboration between language technologists and historical ecologists to slice and dice part of the Dutch National Library's newspaper corpus for the study of interactions between humans and animals. For historical ecology, this approach is challenging: not only because the data is quite unknown territory, but also because studying this material calls for an interdisciplinary approach. The latter is not always straightforward to accomplish. In this project, we have designed a strategy that enables more researchers to make use of the full potential of digital newspapers. 
Recent reintroduction and recolonisation of large mammals and increased competition for space in a multi-use landscape has resulted in higher probabilities of human-animal conflicts. However, where we used to regularly encounter predatory species in the Netherlands (e.g. wolves) or venomous species (e.g. vipers), now encounters with these species are quite rare. Understanding past human-animal conflicts can shed light on the type of conflicts to be expected and how to resolve or even prevent them. It is therefore important to characterise these past relations. How were these animals perceived and why? Were the perceived threats justified? How did people and animals cope with these (adverse) interactions? Answers to these and other questions can help current and future management and policy with regards to human-animal interactions [12].

Digital newspapers archives form an excellent basis to start a comprehensive study on pest and nuisance species. Currently, the majority of such newspaper analyses are done manually, but with the growing size of these collections (the current Dutch National Library's corpus contains 60 million digitised pages), it is increasingly necessary to develop automatic techniques to tackle the timeconsuming task of data collection and filtering according to their relevance to historical ecology research, allowing the researcher to focus on in-depth analyses.

In this paper, we investigate how language technology tools and resources can be ported to the historical ecology domain, which presents interesting challenges: off the shelf state-of-the-art topic classification turned out to be not very suitable for the historical ecology domain and part-of-speech taggers choke on OCR errors. Therefore, the choice was made to focus on investigating a custom, but efficient approach to article classification. The goal of this research is not to develop a new algorithm or system. Instead, our contribution lies in the development of a strategy for the application of state-of-the-art language technology to new domains and in closing the gap between the digital and the humanities.

The contributions of this work are threefold: (1) an annotated research dataset for Dutch historical ecology research, (2) enriched dialect lexicon, (3) a workflow and software for humanities and social sciences researchers to port newspaper classification to their domain.

The remainder of this paper is organised as follows. Background and related work are described in Sect. 2, followed by a description of our data in Sect. 3. Our experimental setup and experiments are presented in Sects. 4 and 5 respectively. We discuss a deep reading case using our data, as well as the strengths and weaknesses of our approach in Sect. 6 and end with conclusions and pointers for future work in Sect. 7. Our code and experiments are available at https://github. com/clariah/serpens. ${ }^{1}$

\section{Background and Related Work}

The field of historical ecology studies the interactions between humans and their environment over long-term periods of time. Research programs in this field share

\footnotetext{
${ }^{1}$ Due to copyright restrictions it is not possible to include the article texts, but the articles are freely accessible through the Dutch National Library newspaper portal.
} 
the belief that understanding present ecological conditions requires knowledge on past interactions with human societies [2]. Topics range from documenting changes in population abundances and ecosystem functioning to studying human perception of animals [16]. Historical ecologists commonly rely on a diverse set of sources, including physical evidence (e.g. archaeological remains, pollen records and genetic analyses), but also on more typical humanities and social sciences sources (e.g. maps, written anecdotes, oral histories, photographs, newspapers and restaurant menus) [11]. Digital newspapers collections are a relatively new source in historical ecology and can be used to document the decline of burbot [4], study narratives on the wolf [7] and badger [19], analyse viper bites in a socioeconomic perspective [12], and record catch rates of the Australian snapper [21].

In the past, newspaper analyses were hampered by limited accessibility to newspapers collections. Major digitisation projects have made many collections available to researchers, but the breadth and depth of these digital collections are both a blessing and a curse. For example, searching for vernacular names of animals typically leads to an overwhelming number of results, which are not always relevant to the research question because animal names are used in proverbs and sayings or as surnames. Search interfaces for these collections do not offer solutions for these problems, preventing researchers from focusing on a deeper analysis of the relevant articles. This results in the majority of newspaper analyses currently being done manually which is extremely time consuming.

Apart from dealing with a considerable amount of irrelevant results, the historical language barrier further challenges to retrieval of correct results. Dealing with historical Dutch means dealing with a great variety in spelling and with animal names changing over time. Query expansion based on spelling and dialect variations can mitigate some of these problems [9].

Classification of newspaper articles is a core task in information retrieval, benefiting a wide range of stakeholders such as news aggregators [10] and investment intelligence [20]. Many approaches use some variant of document classification as described in [15, Chap. 13]. Walma [22] presents manual classification of the different meanings of 'morphine' in the Dutch National Library's newspaper collection, and highlights the problems which are similar to our use case. She argues that crowdsourcing and further specification of the article category in the corpus (for example to feuilleton, letters and news) would be helpful. While this may help with some of the filtering, it cannot be used to classify an article topic, or mitigate the recall issue. The Dutch National Library is continually improving its collection, but there are many dimensions to article classification (cf. [13]) such that efficient domain adaptations such as the one described in this paper will remain necessary in the near future.

\section{Data}

In our work, we rely on both structured and unstructured resources. Structured resources include lexicons and thesauri containing domain knowledge. In this section, we further describe the resources that we use. 


\subsection{Unstructured Data: National Library Newspaper Corpus}

The original texts from 1.3 million newspapers, 1.5 million magazine pages and 320,000 books from the 15 th to the 21 st century have been made available by the Dutch National Library through the Delpher portal. ${ }^{2}$

For two reasons, the data used in this research is scoped to newspaper articles published between 1800 and 1940. This scoping is motivated by (1) The OCR quality on these is most likely better than on the older material and (2) this period also saw the "biological reveil", a reawakening of interest in biological, in the Netherlands, which also may be reflected in mentions of animals in newspapers [3].

\subsection{Structured Data: Taxonomic Resources and Lexicons}

From the ATHENA project, ${ }^{3}$ we use a list of pest and nuisance species. For each species, this list provides its Latin name and its common vernacular name. Due to the local and temporal variance in animal names we also employ diachronic lexicons that each contain Dutch language variations across time and dialects ${ }^{4,5}$ [14].

The main source of information is the DIAMANT lexicon [6], which contains both spelling variations (e.g. bonsinc, buntsems, bunzing, bunsing, bonsingen, bunsingen, bonksems, bonghsen, bonsens, bontsinck, bonzing for bunzing and (near-)synonyms (ulk, ulling), both obtained by semi-automatic analysis and manual correction of information from the Dictionary of Middle Dutch (MNW) and the Dictionary of the Dutch Language $(W N T)^{6}$. In the work done for this paper, the lexicon was expanded with links between the historical dictionary entries and the Dutch Species Register ${ }^{7}$ thus enabling the expansion of variant forms from a canonical designation of a species. The Dutch historical word form lexicons for OCR and OCR postcorrection ${ }^{8}$ have been used to estimate OCR quality.

\subsection{OCR Quality}

While the Delpher newspaper data was digitised and OCR'ed relatively recently, the OCR quality is not consistent and of perfect quality. Most OCR software will give an indication of the certainty of its decisions by attaching a score to a document or batch of documents. However, these scores often give an indication of the errors at the character level, while for our purpose, it is more useful to know how many words (or tokens) are correct in a text, as information extraction techniques do not read as easily over character errors as humans do. Moreover,

2 http://www.delpher.nl.

3 http://www.athena-research.org/.

${ }^{4}$ http://ivdnt.org/onderzoek-a-onderwijs/projecten/gigant.

${ }^{5}$ http://ivdnt.org/onderzoek-a-onderwijs/projecten/diamant.

${ }^{6}$ http://gtb.ivdnt.org.

7 https://www.nederlandsesoorten.nl/.

${ }^{8}$ https://ivdnt.org/downloads/taalmaterialen/tstc-int-historische-woordenlijst. 
this information was not accessible in the downloaded articles. To get an indication of the OCR quality, we developed a lexicon-based OCR quality check. This method checks what proportion of tokens present in an article occurs in the Dutch historical lexicons developed by INT.

Figure 1 shows the results of this measure per 10-year interval. Fortunately, the majority of the texts scores about $70 \%$, although there is some difference between the different time periods. The scores are also provided with our annotated dataset, such that researchers can choose to exclude articles with a lower OCR score.

The OCR quality measure was not always helpful. We calculated the OCR quality for the entire article but noticed that a high scoring article does not always mean that the quality of the context (snippets) of our search term is equally high. Hence the annotation 'Bad OCR' is still possible for an occurrence in an article deemed of good OCR quality according to the lexicon coverage.

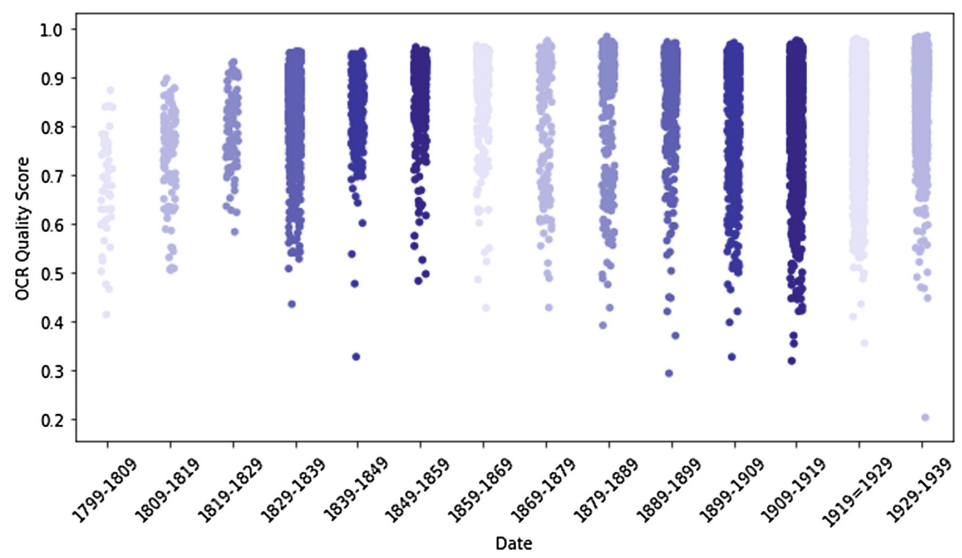

Fig. 1. Lexicon-based OCR check on annotated data

\subsection{Data Categories}

In [8], we developed nine document categories for the natural history domain. These categories are based on analysis of 8,045 manually annotated articles from the Dutch National Library's newspaper corpus in which the term 'bunzing' (European polecat) or 'lynx' occurred. The categories were chosen based on a combination of practicality and research interests. The most important categorization is the distinction between 'animal' and 'no beast'. The 'animal' category is subdivided in finer categories more driven by the research questions. First, there is the distinction of 'figurative' use of the word, such use may provide a general sense of perception. Then there are the categories reporting on real life human-animal interaction. These categories can be typed by sentiment, i.e. 
positive ('natural history'), neutral ('hunt for economic reasons', 'accidents' and 'other') and negative ('nuisance material', 'nuisance immaterial' and 'pest control'). The categories will, in first place, be used as filters for more detailed qualitative analysis. A secondary use case is quantitative, for example, for analysing the number of mentions of organised hunting parties through time. Furthermore, an additional category 'bad OCR' was added. This category is not specific to the ecology domain, but an artifact of the newspaper digitisation process where the article was illegible and the annotator could not decide to which category to assign the article. This results in the following categories:

Natural history. General articles about the animal, e.g. it subsists on birds or $\mathrm{x}$ number were stuffed and became part of a museum collection

Nuisance, material damages. The article mentions the animal as causing material damages, e.g. beetles damaging crops or lynxes killing chickens

Nuisance, immaterial damages. The article mentions the animal as a nuisance without material damages e.g. polecats found to walk over someone's face whilst they were in bed, or (possibly irrational) fear for a certain animal

Pest control. Organised hunt to bring down the number of pest species, e.g. ad for hunting dogs

Hunt for economic reasons. Hunting to use the fur, meat or other parts of the animal e.g. an article mentioning that the hunting season has started again

Prevention. Non-lethal actions against pest species, e.g. advice in the newspaper on which plants keep away pest species. As there was only one article annotated in this category, it was excluded from the classification experiments.

Accidents. Mention of an unintentional encounter with the animal, e.g. roadkill Figurative. Figurative language featuring the animal e.g. eyes like a lynx

Other beast. Mentions of animals in other settings e.g. drawings or religion

No beast. Articles not pertaining to the animal, e.g. a ship named 'Lynx' or a person whose last name is 'Bunzing'

Bad OCR Illegible text due to an abundance of OCR errors e.g. "illlllllliiiiliilitlll" instead of "Best Broadcast Seeder in the World".

\section{Experimental Setup}

We carried out a series of experiments to investigate an optimal balance between annotation effort and performance. The motivation for this is to investigate the minimal annotation effort needed across different animal species in order to automate as much of the data preparation for historical ecologists. Additionally, our findings may be ported to other domains where there is a clear information need and little training data available (e.g. medicine [22] or criminal acts [1]). Our workflow is presented in Fig. 2.

1. Query construction: Queries are based on the animal taxonomy lists containing known pest and nuisance species compiled by the ATHENA project. ${ }^{9}$

\footnotetext{
9 http://www.athena-research.org/.
} 


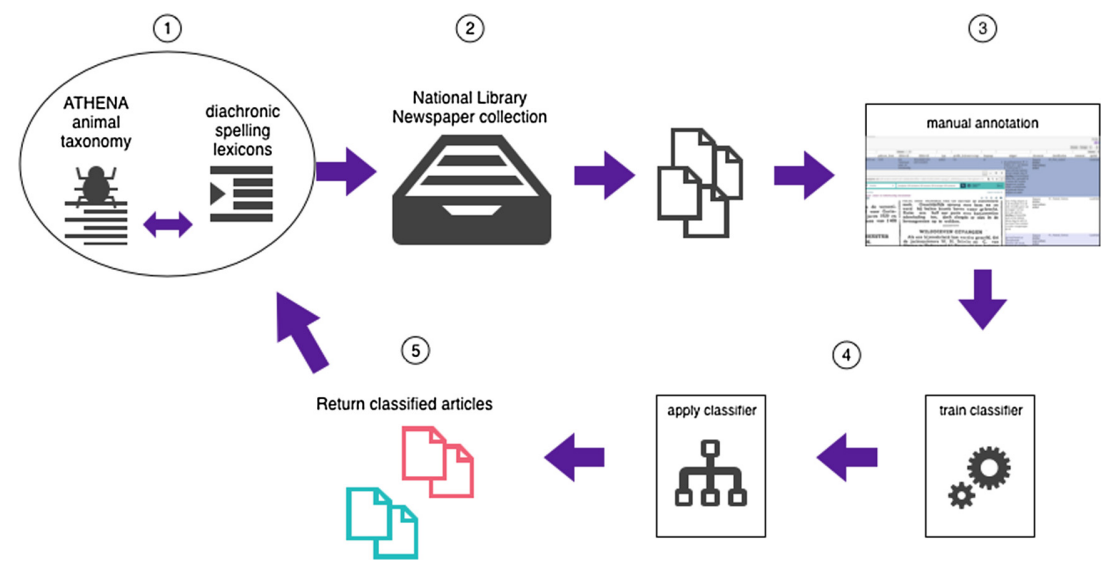

Fig. 2. Experimental setup

These lists contain the species' scientific name as well as the vernacular. From this list, we decided to focus on the mammals that are known pest and nuisance species. We complement this list with spelling variations of the vernacular name from the DIAMANT lexicon (cf. above). As the synonym detection was still partly work in progress when we collected the research data, we focused on spelling variation and inflection. For one species, we also included a synonym and its spelling variations, namely for muskrat / Ondatra zibethicus. We queried for both 'muskusrat' and 'bisamrat' as these are both very common. Upon inspection of the newspaper corpus, we find that 'bisamrat' is only used after 1910 in our corpus, and 'muskusrat' occurs throughout. ${ }^{10}$

2. Article retrieval: The National Library's API was used to retrieve the OCR'ed text for each newspaper article matching the query as well as the article's metadata. This resulted in a total of 25,400 articles for the 8 performed queries.

3. Manual annotation: 9,931 Articles were annotated manually by a historical ecologist and a lexicologist through the annotation environment shown in Fig. 3. In this environment, the annotator is shown a snippet of the article around the query match. If necessary s/he can choose to see the full text of the article or the scanned image of the article. Annotation is done by selecting a category from a fixed list. In this environment, annotating 1,000 articles took 4 to $5 \mathrm{hrs}$. on average. Statistics on the annotated articles are presented in Table 1.

4. Train and test classifier: We train and test various classifiers on the dataset, as well as with varying amounts of training data to find a sweet spot of good results with a low annotation load. The test scenarios are further detailed in Sect. 5

$\overline{10}$ https://www.delpher.nl/nl/kranten/ngram. 
5. Return documents: The classified documents are returned to the user and the enrichments are fed back to the ATHENA Research portal as well as the CLARIAH Timbuctoo research data portal. ${ }^{11}$ Furthermore, information from the classified documents is fed back to the DIAMANT lexicon. Currently, we do not detect new variants or species names, but we to provide additional word senses and attestations of the already known species names, thus enriching semantic information in the lexicon and the information on the diachronic distribution of terms. The combination of the time stamp of the article and the classification were used to select either quotations with a more recent date than present in the entry describing an animal, or to track potential meanings not yet described. For the latter, bunzing (polecat) is a good example, since it lacked the meaning "fur" and "piece of cloathing made of polecat fur."

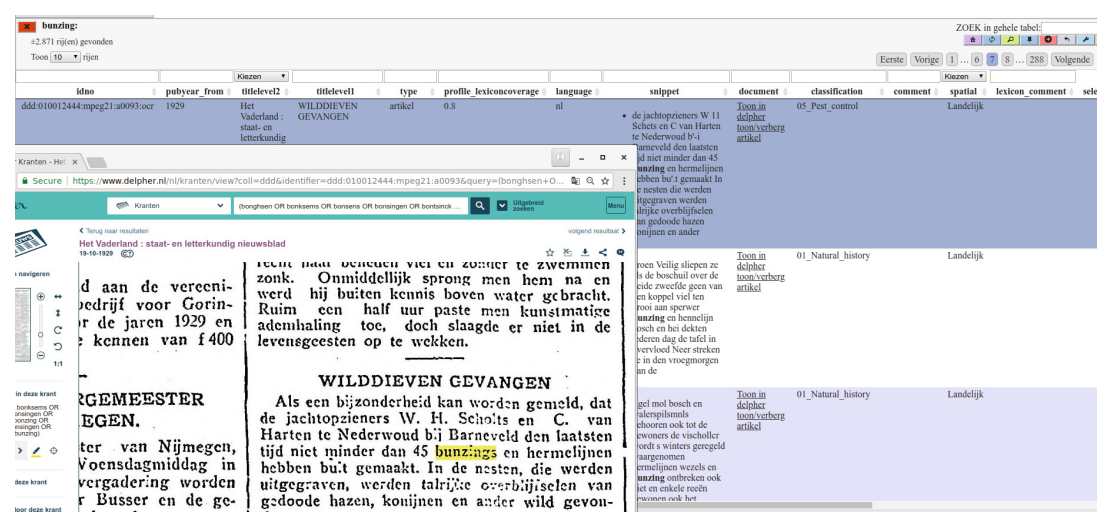

Fig. 3. Screen shot of the SERPENS annotation environment

\section{$5 \quad$ Experiments and Results}

In our experiments, all texts are preprocessed as following: the mentions of the animal name are substituted by the value $=A N I M A L=$. This is to prevent the classifier from classifying based on the species name, rather than the context in which it occurs. There are for example class imbalances as we saw that the retrieved articles for 'bunzing' (European polecat) are more often about the animal than for example the articles retrieved for 'lynx'. The texts are then transformed into a bag of words representation and normalised using tf.idf [15, Chap. 6].

We first perform an exploratory 10-fold cross validation experiment on the entire dataset to gain insights into which classifier is most promising. Table 2

${ }^{11}$ https://timbuctoo.huygens.knaw.nl/. 
Table 1. Dataset statistics with NH (natural history), NM (nuisance material), NI (nuisance immaterial), PC (pest control), H (hunt), A (accidents), F (figurative), O (other), and BO (bad OCR)

\begin{tabular}{|c|c|c|c|c|c|c|c|c|c|c|c|c|}
\hline Vernacular & $\begin{array}{l}\text { Taxonomic } \\
\text { name }\end{array}$ & Total & $\mathrm{NH}$ & $\mathrm{NM}$ & NI & $\mathrm{PC}$ & $\mathrm{H}$ & $\mathrm{A}$ & $\mathrm{F}$ & $\mathrm{O}$ & NB & $\mathrm{BO}$ \\
\hline $\begin{array}{l}\text { Beverrat } \\
\text { (coypu) }\end{array}$ & $\begin{array}{l}\text { Myocastor } \\
\text { coypus }\end{array}$ & 51 & 31 & - & - & 14 & 6 & - & - & - & - & - \\
\hline $\begin{array}{l}\text { Bisamrat } \\
\text { (muskrat) }\end{array}$ & $\begin{array}{l}\text { Ondatra } \\
\text { zibethicus }\end{array}$ & 238 & 42 & 6 & 10 & 109 & 61 & - & 2 & 8 & - & - \\
\hline $\begin{array}{l}\text { Boommarter } \\
\text { (European } \\
\text { pine marten) }\end{array}$ & Martes martes & 135 & 45 & 3 & - & 47 & 37 & 1 & 2 & - & - & - \\
\hline $\begin{array}{l}\text { bunzing } \\
\text { (European } \\
\text { polecat) }\end{array}$ & $\begin{array}{l}\text { Mustela } \\
\text { putorius }\end{array}$ & 2833 & 636 & 356 & 95 & 472 & 734 & 229 & 119 & 14 & 166 & 12 \\
\hline $\begin{array}{l}\text { Hermelijn } \\
\text { (stoat) }\end{array}$ & $\begin{array}{l}\text { Mustela } \\
\text { erminea }\end{array}$ & 395 & 10 & 1 & - & 11 & 364 & - & 5 & - & 3 & 1 \\
\hline $\operatorname{lynx}(\operatorname{lynx})$ & Lynx lynx & 5,491 & 325 & 16 & 5 & 4 & 316 & - & 205 & - & 4620 & - \\
\hline $\begin{array}{l}\text { Nerts } \\
\text { (European } \\
\text { mink) }\end{array}$ & Mustela lutreola & 296 & - & 1 & - & - & 34 & - & 1 & - & 46 & 214 \\
\hline $\begin{array}{l}\text { Steenmarter } \\
\text { (beech } \\
\text { marten) }\end{array}$ & Martes foina & 501 & 56 & 5 & - & 16 & 397 & - & - & - & 13 & 14 \\
\hline \multicolumn{2}{|l|}{ Total } & 9,940 & 1,144 & 388 & 110 & 669 & 1,947 & 230 & 334 & 22 & 4,848 & 239 \\
\hline
\end{tabular}

shows our baseline experiments. The classifiers we tested are: (1) Decision Tree using Gini impurity to measure the quality of a split and 'best split' at each node. (2) K-nearest neighbors with $\mathrm{k}=3$ and Euclidean distance as the evaluation metric, and brute force search algorithm. (3) A linear Support Vector Machine using Stochastic Gradient Descent training using libsvm (4) Multinomial Naive Bayes and (5) Linear Support Vector Classification using liblinear using the default settings in scikit learn. ${ }^{12}$ The reported metrics are macro averages.

The top half of the table shows the results on the entire newspaper article, the bottom half on only the snippet surrounding the species name that matched the query. As the results indicate, the results on the snippets are slightly better than those on the full texts. We suspect that this is due to the fact that newspaper articles are not always about only one topic. In particular longer articles sometimes discuss animals as well as other issues. ${ }^{13}$

We see here that the Linear Support Vector outperforms the other classifiers. Instead of focusing on further parameter tuning, we delve into the analysis of the text snippets classification and various training/test setups using this classifier.

\footnotetext{
12 http://scikit-learn.org/stable/index.html.

${ }^{13}$ For example https://www.delpher.nl/nl/kranten/view?coll=ddd\&identifier=KBN RC01:000057072:mpeg21:a0081 which is an installment of a translation an Edison Marshall story discussing the trials and tribulations of several travellers, which includes a passage on hunting a muskrat.
} 
Table 2. Results of 10-fold cross-validation experiments using different algorithms

\begin{tabular}{l|l|l|l|l}
\hline Class & Precision & Recall & F $_{1 \text {-score }}$ & Correct \\
\hline Classification on full article text & \multicolumn{5}{|l}{} \\
\hline Decision Tree & 0.648 & 0.646 & 0.647 & 6,413 \\
\hline K-Nearest Neighbours & 0.769 & 0.655 & 0.690 & 6,500 \\
\hline Support Vector Machine & 0.692 & 0.730 & 0.685 & 7,245 \\
\hline Naive Bayes & 0.553 & 0.546 & 0.422 & 5,421 \\
\hline Linear Support Vector Classification & 0.774 & 0.769 & 0.770 & 7,640 \\
\hline Classification on text snippets & & & & \\
\hline Decision Tree & 0.640 & 0.644 & 0.642 & 6,386 \\
\hline K-Nearest Neighbours & 0.794 & 0.694 & 0.720 & 6,876 \\
\hline Support Vector Machine & 0.749 & 0.752 & 0.717 & 7,453 \\
\hline Naive Bayes & 0.743 & 0.754 & 0.711 & 7,472 \\
\hline Linear Support Vector & 0.797 & 0.796 & 0.796 & 7,884 \\
\hline
\end{tabular}

When taking a closer look at the per-class performance of the Linear Support Vector in Table 3, we see that the large classes perform best, which is to be expected. As the 'Bad OCR' category can contain articles that could potentially fall in any of the content categories, it is no surprise that the classifier has difficulties predicting this class. The same holds for 'Other beast', which, on top of its diversity, is also very small (only 22 examples).

The confusion matrix in Fig. 4 provides some more information on which classes the classifier had difficulties with. For example, the classifier has some trouble distinguishing between 'Pest control' and 'Hunt for economic reasons'. This is no surprise, as certain species were both seen as a pest but their fur could still be sold, so the distinction between the classes is not always easy to make. Furthermore, 'Figurative' and 'No beast' also get confused regularly, which can be explained by figurative language use indeed often being about something other than an animal.

As it is most important for researchers to not have to spend time on irrelevant documents, we also ran a series of experiments in which we reduced the categorisation problem to two classes: beast vs no beast. In the 'beast' category, we lumped together the categories 'Natural history', 'Nuisance material', 'Nuisance immaterial', 'Pest control', 'Hunt economical', 'Accidents', and 'Figurative'. Potentially, this could also speed up the initial manual annotation as the annotator would only need to distinguish between two classes.

This results in scores that are going into the $90 \%$ range, as presented in Table 4. For these experiments, we also include the Naive Bayes results, as the performance here is approaching that of the Linear Support Vector classifier. The class balance (nearly 50-50) is probably also helping the classifiers here.

For these experiments, we annotated nearly 10,000 examples. Whilst this is a useful exercise to get to know the data, this is not desirable to repeat for every 
Table 3. Per-class results of 10 -fold cross validation experiments using a linear support vector model

\begin{tabular}{l|l|l|l|l}
\hline Class & Precision & Recall & $F_{1 \text {-score }}$ & Correct \\
\hline Natural history & 0.644 & 0.725 & 0.682 & 829 \\
\hline Nuisance material & 0.599 & 0.639 & 0.618 & 248 \\
\hline Nuisance immaterial & 0.500 & 0.464 & 0.481 & 51 \\
\hline Pest control & 0.471 & 0.462 & 0.466 & 309 \\
\hline Hunt economical & 0.866 & 0.831 & 0.848 & 1618 \\
\hline Accidents & 0.602 & 0.552 & 0.576 & 127 \\
\hline Figurative & 0.581 & 0.656 & 0.616 & 219 \\
\hline Other beast & 0.143 & 0.045 & 0.069 & 1 \\
\hline Nobeast & 0.923 & 0.913 & 0.918 & 4426 \\
\hline Bad OCR & 0.293 & 0.256 & 0.273 & 56 \\
\hline Avg/Total & 0.797 & 0.796 & 0.796 & 7,884 \\
\hline
\end{tabular}

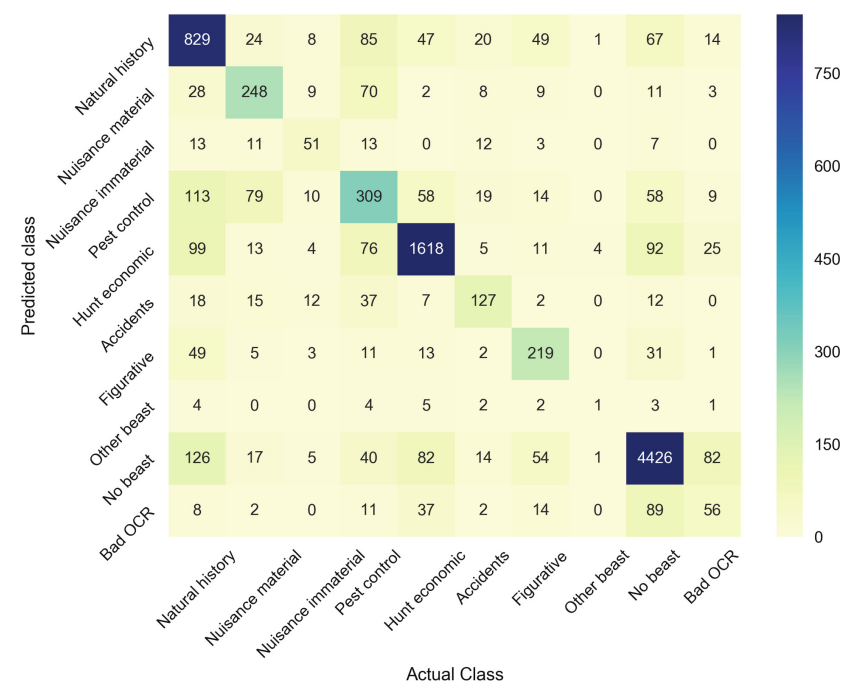

Fig. 4. Confusion matrix 10-fold cross-validation linear support vector

Table 4. Results of 10-fold cross validation experiments two classes

\begin{tabular}{l|l|l|l|l|l|l|l|l}
\hline & \multicolumn{4}{|l}{ Linear support vector } & \multicolumn{4}{l}{ Naive Bayes } \\
\hline Class & Precision & Recall & F1-score $_{1}$ & Correct & Precision & Recall & F $_{1 \text {-score }}$ & Correct \\
\hline Beast & 0.919 & 0.928 & 0.924 & 4,453 & 0.880 & 0.974 & 0.925 & 4,719 \\
\hline No beast & 0.928 & 0.919 & 0.923 & 4,497 & 0.971 & 0.867 & 0.916 & 4,202 \\
\hline Avg/Total & 0.924 & 0.924 & 0.924 & 8,950 & 0.925 & 0.921 & 0.920 & 8,921 \\
\hline
\end{tabular}


domain. We therefore investigate the bias and variance via the learning curves presented in Fig. 5. On the left-hand side, we have plotted the learning curves for the all classes experiments using the snippets and the Linear Support Vector classifier and Naive Bayes, on the right-hand side, for the two-class classification setup as described in Table 4.

As Fig. 5b and d illustrate, for the two-class setup, an increase in training data quickly leads to very little improvement of the results. Even only $\sim 1,000$ annotated examples show a potential to obtain a good classification.

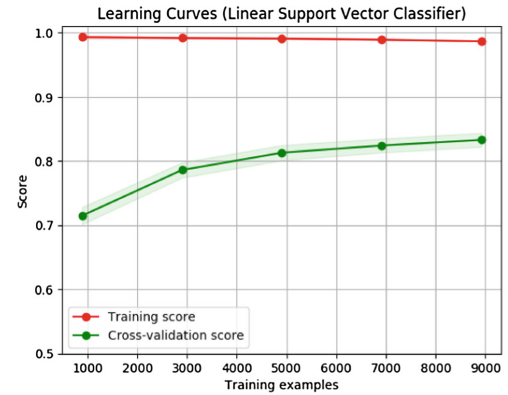

(a) All classes SVM

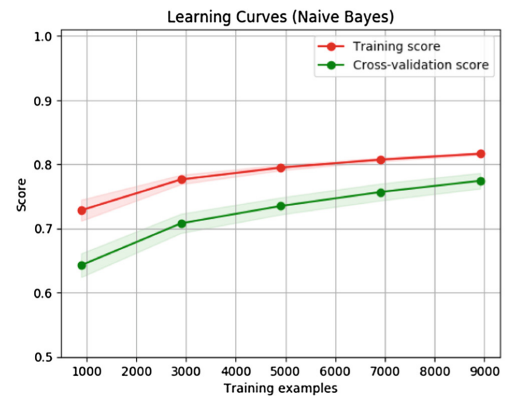

(c) All classes Naive Bayes

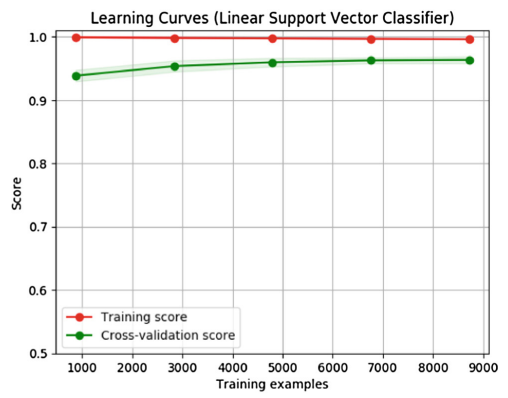

(b) Animal vs. no animal SVM

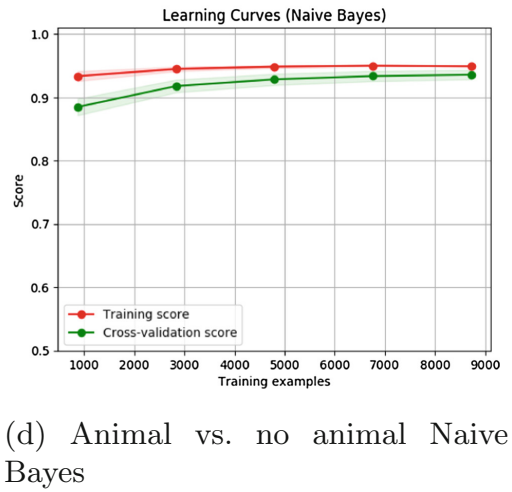

Fig. 5. Learning curves for all classes and animal vs no animal classification

\section{Discussion}

We have started using the created dataset to study the impact of different pest and nuisance species on human practices and how the public perception of these animals has changed. We show an example in Fig. 6. In this figure, an example of distant reading [17], the class distribution of the data for European polecats (bunzing) is plotted per 10-year interval. This can be used to identify interesting 
periods to investigate when the researcher is for example looking for mentions of animals in the context of hunt for economic reasons (1910-1919 would be a good candidatem 1880-1889 less so). It should be noted that there are gaps in the digitisation of the newspapers and some articles may be published by several newspapers, therefore such analyses should also be seen in the context of the total corpus distribution and combined with close reading analyses.

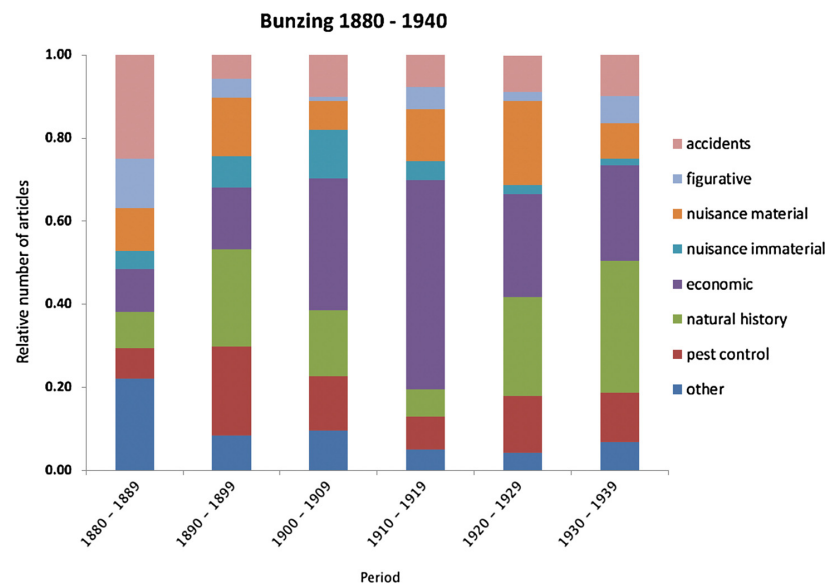

Fig. 6. Distant reading of European Polecat data

As mentioned in Sect. 3, the OCR quality of the corpus remains an issue for improvement. We now measured OCR quality on the entire article, whereas it may also be useful to focus it on the snippets around the keyword, which were also used for further classification.

When working on a particular use case on a longer term, further experiments could include parameter tuning.

\section{Conclusions and Future Work}

We have presented a case study on porting language technology to the historical ecology domain. Our experiments show that even $\sim 1,000$ examples can yield a good basis for distinguishing relevant from irrelevant newspaper articles, automating a major data filtering task that had impeded large scale historical ecology studies thus far.

With nearly 10,000 examples $\mathrm{F}_{1}$ of up to 0.92 are obtained. The annotated dataset that we have created in the course of this research is available for researchers, and it has already been used by the lexicologists on this project to extend their lexicons, indicating that interdisciplinary research is a mutually beneficial undertaking. 
Furthermore, our workflow can be adopted by other domains to bootstrap their data filtering problem, although we recommend performing such experiments in an interdisciplinary setting as different domains and datasets may still need some adaptations. We are working with a team of social economic historians to adopt this workflow in a project on diamonds as commodities. ${ }^{14}$

We will further expand the lexicons with "folk names", using dialect dictionaries and popular books on animals to increase the coverage of the lexicons, in particular for regional newspapers.

Our presented classification may also be complemented by an unsupervised method, such as unsupervised topic modelling or clustering. This may help us identify more articles concerning 'Pest control' regardless of the species name. This is something to be investigated in future work as well as multilabel classification. The National Library's newspaper corpus is already annotated with broad article categories such as 'advertisement', 'article' and 'family announcement' but a further classification such as 'news', 'feuilleton' and 'letter' (as also suggested by [22]) may help improve filtering.

As some of the relevant classes are difficult to predict using only the text, we are investigating further analysis of the articles and their text features. Some of the animal names are quite polysemous (e.g. 'das', which can mean 'badger', 'scarf' or 'tie', it's the name of a beer brand as well as of an insurance company and has several figurative uses) therefore additional information from for example word sense disambiguation may be helpful.

Our use case presents one step into the direction of closing the gap between the digital and the humanities.

Acknowledgments. The research for this paper was made possible by the CLARIAHCORE project financed by NWO: http://www.clariah.nl. We thank the Dutch National Library for providing access to their newspaper corpus.

\section{References}

1. Arulanandam, R., Savarimuthu, B.T.R., Purvis, M.A.: Extracting crime information from online newspaper articles. In: Proceedings of the Second Australasian Web Conference-Volume 155, pp. 31-38. Australian Computer Society, Inc. (2014)

2. Balée, W.: The research program of historical ecology. Annu. Rev. Anthropol. 35, 75-98 (2006)

3. van Berkel, K.: Vóór Heimans en Thijsse: Frederik van Eeden sr. en de natuurbeleving in negentiende-eeuws Nederland, vol. 63. Koninklijke Nederlandse Akademie van Wetenschappen (2006)

4. Bosveld, J., Kranenbarg, J., Lenders, H., Hendriks, J.: Historic decline and recent increase of burbot, in the Netherlands. Hydrobiologia 757

5. Brukner, P., Gara, T.J., Fortington, L.V.: Traumatic cricket-related fatalities in australia: a historical review of media reports. Med. J. Aust. 208(6), 261-264 (2018)

$\overline{14}$ https://www.clariah.nl/projecten/research-pilots/db-ccc. 
6. Depuydt, K., de Does, J.: The diachronic semantic lexicon of dutch as linked open data. In: Proceedings of the Eleventh International Conference on Language Resources and Evaluation (LREC 2018). European Language Resources Association (ELRA), Paris, France, May 2018

7. Dirke, K.: Where is the big bad wolf? Notes and narratives on wolves in swedish newspapers during the eighteenth and nineteenth centuries. In: Masius, P., Sprenger, J. (eds.) A Fairy Tale in Question. Historical Interactions Between Humans and Wolves, pp. 101-118. The White Horse Press, Cambridge (2015)

8. van Erp, M., van Goethem, T., Depuydt, K., de Does, J.: Towards semantic enrichment of newspapers: a historical ecology use case. In: Proceedings of the Second Workshop on Humanities in the Semantic Web (WHiSe II) Co-located with 16th International Semantic Web Conference (ISWC 2017), Vienna, Austria, 22 October 2017. http://ceur-ws.org/Vol-2014/paper-05.pdf

9. Gotscharek, A., Reffle, U., Ringlstetter, C., Schulz, K.U., Neumann, A.: Towards information retrieval on historical document collections: the role of matching procedures and special lexica. IJDAR 14(2), 159-171 (2011)

10. Koperski, K., Bhatti, S., Liang, J., Klein, A.: Cluster-based identification of news stories, August 25 2015, uS Patent 9,116,995

11. Kwok, R.: Historical data: hidden in the past. Nature 549, 419-421 (2017)

12. Lenders, H.J.R.: Ten a penny? Deadly viper bites in the netherlands in a socioeconomic perspective. Litteratura Serpentium 34, 290-316 (2014)

13. Lonij, J., Harbers, F.: Genre classifier (2016). http://lab.kb.nl/tool/genre-classifier

14. Maks, I., van Erp, M., Vossen, P., Hoekstra, R., van der Sijs, N.: Integrating diachronous conceptual lexicons through linked open data. Presented at DHBenelux 2016, 9-10 June 2016

15. Manning, C.D., Raghavan, P., Schütze, H.: Introduction to Information Retrieval. Cambridge University Press, Cambridge (2008)

16. Mcclenachan, L., Cooper, A., McKenzie, M., Drew, J.: The importance of surprising results and best practices in historical ecology. BioScience 65 (2015). https:// doi.org/10.1093/biosci/biv100

17. Moretti, F.: Distant Reading. Verso Books (2013)

18. Nerghes, A., Hellsten, I., Groenewegen, P.: A toxic crisis: metaphorizing the financial crisis. Int. J. Commun. 9, 27 (2015)

19. Runhaar, H., Runhaar, M., Vink, H.: Reports on badgers meles meles in Dutch newspapers 1900-2013: same animals, different framings? Mammal Rev. 45(3), 133-145 (2015)

20. Seo, Y.W., Giampapa, J.A., Sycara, K.: Financial news analysis for intelligent portfolio management. Technical report. CMU-RI-TR-04-04, Carnegie Mellon University (2004)

21. Thurstan, R., Campbell, A., Pandolfi, J.: Nineteenth century narratives reveal historic catch rates for australian snapper (pagrus auratus). Fish Fish. 17, 210225 (2016)

22. Walma, L.: Filtering the 'news': uncovering Morphine's multiple meanings on Delpher's Dutch newspapers and the need to distinguish more article types. TS: Tijdschrift voor Tijdschriftstudies 38, 61-78 (2015)

23. Yzaguirre, A., Smit, M., Warren, R.: Newspaper archives + text mining $=$ rich sources of historical geo-spatial data. IOP Conf. Ser. Earth Environ. Sci. 34(1), 012043 (2016) 